
\title{
BReserch S Suare \\ Sex Differences In Impact of Sarcopenia On Falls In Community-Dwelling Older Adults
}

\section{Chang Won Won}

Kyung Hee University Medical Center

Yunsoo Soh ( $\nabla$ soyuns@gmail.com )

Kyung Hee University Medical Center

\section{Research Article}

Keywords: aging, sarcopenia, falls, Asian Working Group for Sarcopenia, body composition

Posted Date: September 21st, 2021

DOl: https://doi.org/10.21203/rs.3.rs-885429/v1

License: (c) (i) This work is licensed under a Creative Commons Attribution 4.0 International License. Read Full License 


\section{Abstract}

Background: Falls are one of the most serious health problems among older adults. Sarcopenia is characterized by a decrease in muscle mass, strength, and physical function. Due to potentially agerelated conditions, both falls and sarcopenia have common risk factors. However, the association between sarcopenia and falls is controversial.

Methods: In this cross-sectional study, we used data from the Korean Frailty and Aging Cohort Study; 2,323 community-dwelling older adults (1,111 men and 1,212 women) aged 70-84 years were recruited in this cross-sectional study. To evaluate sarcopenia, the Asian Working Group for Sarcopenia (AWGS) diagnostic algorithm was used. We compared the faller and non-faller groups. Unadjusted and fully adjusted logistic regression analyses were performed to evaluate the relationship between sarcopenia and falls.

Results: A total of 239 (24.1\%) women in the faller group had a history of falls in the past year, which was statistically higher than that in men $(176,15.8 \%)$. In the fully adjusted model, handgrip strength (odds ratio $[\mathrm{OR}]=1.508,95 \%$ confidence interval $[\mathrm{Cl}]=1.028-2.211)$, short physical performance battery $(\mathrm{OR}=2.068,95 \% \mathrm{Cl}=1.308-3.271)$, and 4-m walking speed (OR=3.499, 95\% $\mathrm{Cl}=2.044-5.991)$ were significantly lower in the male faller group. However, the female faller group only showed a significantly low appendicular skeletal muscle mass index $(\mathrm{OR}=1.419,95 \% \mathrm{Cl}=1.058-1.903)$ in the fully adjusted model.

Conclusions: This large cohort study aimed to identify the incidence of sarcopenia in older Korean population using the AWGS diagnostic algorithm and its correlation with falls. The incidence of falls did not increase in the sarcopenia group. Among the sarcopenia components, sex differences affect the a history of falls. Therefore, when studying the risk of falls in old age, sex differences should be considered.

\section{Background}

Falls are one of the most serious problems that threaten the health of older adults and untimely cause death, physical injury, immobility, socio-psychological dysfunction, and hospitalization [1]. Falls can be caused by various factors, including aging, female sex, living alone, cognitive impairment, fear of falling, vitamin D deficiency, multiple medications, visual impairment, and chronic comorbidities such as cardiopulmonary disease, depression, osteoarthritis, and diabetes [2, 3]. The risk of falls increases dramatically in people aged $>75$ years. In the community-dwelling population, one-third of older adults aged $>65$ years and $50 \%$ individuals aged $>80$ years experience falls each year; among them, $>40 \%$ experience recurrence [4]. Approximately $20-30 \%$ of fallers sustain fall-related injuries that reduce their mobility and independence in performing the activities of daily living and increase the risk of premature death [5].

The physiological changes that usually occur due to aging vary, and one of them is sarcopenia, caused by a decrease in skeletal muscle mass. Sarcopenia directly causes a decrease in muscle strength and 
physical functions, thus causing disability and increasing the risk of mortality [6, 7]. Muscle mass and strength decrease as a person ages, and changes in body composition occur in both men and women; these changes occur regardless of the changes in body weight [8]. The prevalence of sarcopenia differs depending on the diagnostic method used and sex; sarcopenia occurs in $10 \%$ individuals aged $>65$ years, and its prevalence increases with age by $>50 \%$ after the age of 80 years [9]. The factors related to the development of sarcopenia include reduction in muscle cells; imbalance between protein breakdown and synthesis; decrease in the levels of inflammatory cytokines, cortisol, and sex hormones; insulin resistance; and lifestyle-related factors such as nutritional intake and physical activity. In particular, lifestyle-related factors are the primary risk factors for sarcopenia, including malnutrition, reduced physical activity, alcohol intake, and smoking [10]. As aging reduces the skeletal muscle mass, muscle strength, endurance, and contractility are affected, and an individual's sense of balance decreases. Falls are a serious health problem among older adults. Moreover, this decrease in proprioceptive sensory function leads to a decrease in the ability to balance, which causes difficulties in motor control. Changes in these factors lead to a decrease in physical function and slowness in gait, thus increasing the risk of falls in older adults [11].

In 2010, the European Working Group on Sarcopenia in Older People (EWGSOP) proposed a diagnostic algorithm for sarcopenia, which was revised in 2019 as ESGSOP2 [12]. Because of the relatively smaller body size, higher adiposity, and less mechanized, more physical activity among the Asian population, the Asian Working Group for Sarcopenia (AWGS) updated the diagnostic algorithm in 2019 based on Asian data [13]. Sarcopenia as a risk factor for falls in older adults is controversial. This study aimed to investigate the impact of sarcopenia, defined by the AWGS, on falls in community-dwelling Korean older adults using baseline data from the Korean Frailty and Aging Cohort Study (KFACS).

\section{Methods}

\section{Data and study population}

In this cross-sectional study, we used the data from the 2016-2017 KFACS to investigate the relationship between sarcopenia, defined by the AWGS, and falls. The KFACS is a nationwide, multicenter study performed in eight medical and two public health centers across South Korea in community-dwelling older adults aged 70-84 years. A total of 3,014 participants with incomplete data on weakness due to cerebrovascular accident, with a deformity or motor deficit in the extremities, with severe cognitive impairment and blindness, with inability to complete the physical performance test including the 4-m walking speed, who did not undergo short physical performance battery (SPPB) test, and without DEXA data were excluded. Finally, 2,323 participants (1,111 men and 1,212 women) were included (Fig. 1). Data on baseline demographics and medical history, including age, sex, years of education ( $<6$ years, 6-12 years, or $>12$ years), location of residence (rural or city), smoking status, alcohol consumption, body mass index (BMI), and chronic comorbidities, were also collected. Participants who smoked more than one cigarette per week and drank alcohol at least once a week were defined as smokers and drinkers, respectively. The KFACS protocol was approved by the Institutional Review Board (IRB) of the Clinical 
Research Ethics Committee of Kyung Hee University Medical Center (IRB number: 2015-12-103), and all participants provided written informed consent.

\section{Sarcopenia}

Sarcopenia was defined using the AWGS diagnostic criteria, which was updated in 2019 [13]. Participants with both low muscle strength and low muscle mass were included in the sarcopenia group. Participants in the sarcopenia group with low physical performance (SPPB score: < 9 points) were further categorized into the severe sarcopenia group.

1. Muscle strength: Using a hand dynamometer (JAMAR, Bolingbrook, IL, USA), the participants' handgrip strength (HGS) on both sides was measured twice, with the elbow flexed at $90^{\circ}$ while in a sitting position, and the highest value was obtained (cutoff values: $<28 \mathrm{~kg}$ for men and $<18 \mathrm{~kg}$ for women).

2. Muscle mass: DEXA is more widely used as it is a noninvasive, accurate, and convenient method for measuring muscle quantity [14]. Using DEXA, we calculated the appendicular skeletal muscle mass index (ASMI) at different heights, and the results are expressed as ASM/height ${ }^{2}$ (cutoff values: $<7.0$ $\mathrm{kg} / \mathrm{m}^{2}$ for men and $<5.4 \mathrm{~kg} / \mathrm{m}^{2}$ for women).

3. Physical performance: The SPPB test is a well-established physical performance test that evaluates the lower extremities in older adults. This test assesses the following items: standing balance, walking speed, and five repeated chair stands. Each item was scored 4 points, with a higher score indicating better lower extremity physical function. In the AWGS diagnostic criteria, a score of $\leq 9$ points indicates low physical performance. The 4-m usual walking speed was measured using an electronic device (cutoff value: $\leq 1.0 \mathrm{~m} / \mathrm{s}$, both sexes)

\section{Falls}

Falls was confirmed based on the participants' response to the following question: In the past year, have you had falls? "Falls" was defined as a sudden contact of any part of the body, except the feet, with the ground. Participants with a history of falls were classified as fallers, while those with no history of falls were classified as non-fallers.

\section{Statistical analysis}

The demographic characteristics of the participants were analyzed using a t-test for continuous variables and the chi-square test for categorical variables. The results are expressed as mean \pm standard deviation (SD) or number and ratio (\%) according to the characteristics of the variables. Unadjusted and fully adjusted analyses were performed using logistic regression models to examine odds ratios (ORs). Each model was fully adjusted for multiple interrelations between sarcopenia and other potential confounding variables such as age, depression, osteoarthritis, osteoporosis, diabetes mellitus, hypertension, location of residence, family group, vitamin D level, number of medications, and BMI. The collected data were 
analyzed using SPSS 23.0 software (IBM, Inc., Chicago, IL), and a P-value of $<0.05$ was considered significant.

\section{Results}

The baseline characteristics of the participants are shown in Table 1. Among the 2,325 participants, 1,111 (47.7\%) were men and 1,212 (52.3\%) were women. A total of $239(24.1 \%)$ women in the faller group had a history of falls in the past year, which was statistically higher than that in men (176, 15.8\%). Sex, age, $\mathrm{BMI}, \mathrm{HGS}$, ASMI, walking speed, and SPPB score were significantly different between the groups. Other socioeconomic characteristics, including years of education, marital status, income, residency, smoking, and chronic comorbidities, were significantly different between the groups. 
Table 1

Baseline characteristics of the participants according to sex

\begin{tabular}{|c|c|c|c|c|}
\hline \multirow[t]{2}{*}{ Characteristic } & & Male & Female & \multirow[t]{2}{*}{$P$} \\
\hline & & $(n=1111)$ & $(n=1212)$ & \\
\hline Age $($ mean $\pm S D)$ & & $76.87 \pm 3.8$ & $76.11 \pm 3.8$ & $<0.001^{\star}$ \\
\hline $\mathrm{BMI}($ mean $\pm \mathrm{SD})$ & & $23.96 \pm 2.9$ & $24.8 \pm 3.0$ & $<0.001^{\star}$ \\
\hline $\mathrm{HGS}, \mathrm{kg}($ mean $\pm \mathrm{SD})$ & & $32.23 \pm 5.8$ & $21.10 \pm 3.9$ & $<0.001^{\star}$ \\
\hline $\mathrm{ASMI}, \mathrm{kg} / \mathrm{m}^{2}($ mean $\pm \mathrm{SD})$ & & $7.04 \pm 0.8$ & $5.83 \pm 0.7$ & $<0.001^{\star}$ \\
\hline Walking speed, $\mathrm{m} / \mathrm{s}($ mean $\pm \mathrm{SD})$ & & $10.9 \pm 3.5$ & $10.4 \pm 3.7$ & $0.002^{\star}$ \\
\hline SPPB total $($ mean \pm SD $)$ & & $11.09 \pm 1.2$ & $10.59 \pm 1.5$ & $<0.001^{\star}$ \\
\hline Fall in past year, $\mathrm{n}(\%)$ & & $176(15.8)$ & $293(24.1)$ & $<0.001^{\star}$ \\
\hline Fall related fracture, $\mathrm{n}(\%)$ & & $21(1.9)$ & $43(3.5)$ & $0.015^{\star}$ \\
\hline Medications, n (mean \pm SD) & & $3.64 \pm 3.0$ & $3.30 \pm 2.6$ & 0.005 \\
\hline \multirow[t]{3}{*}{ Years of education, $\mathrm{n}(\%)$} & $<6$ & $298(26.0)$ & $688(56.8)$ & \multirow[t]{3}{*}{$<0.001^{*}$} \\
\hline & $7-12$ & $473(42.6)$ & $402(33.2)$ & \\
\hline & $>13$ & $349(31.4)$ & $122(10.1)$ & \\
\hline Marital status, n (\%) & Married & $1021(91.9)$ & $788(65.0)$ & $<0.001^{\star}$ \\
\hline \multirow[t]{3}{*}{ Income, million won per month, $\mathrm{n}(\%)^{\dagger}$} & $>3$ & $216(19.4)$ & $225(18.6)$ & \multirow[t]{3}{*}{$<0.001^{\star}$} \\
\hline & $1-3$ & $553(49.8)$ & $447(36.9)$ & \\
\hline & $<1$ & $342(30.8)$ & $540(44.6)$ & \\
\hline \multirow[t]{2}{*}{ Residency, n (\%) } & Urban & $867(77.9)$ & $1029(85.1)$ & \multirow[t]{2}{*}{$<0.001^{\star}$} \\
\hline & Rural & $244(22.1)$ & $183(14.9)$ & \\
\hline Current smoker, n (\%) & & $730(65.7)$ & $22(1.8)$ & $<0.001 *$ \\
\hline Alcohol use, n (\%) & & $661(59.5)$ & $709(58.0)$ & 0.46 \\
\hline Hypertension, $\mathrm{n}(\%)$ & & $595(53.8)$ & $714(58.9)$ & 0.19 \\
\hline
\end{tabular}

Abbreviations: BMI, body mass index; HGS, handgrip strength; ASMI, appendicular skeletal muscle mass index; SPPB, short physical performance battery; OA, osteoarthritis; WBC, white blood cell; $\mathrm{Hb}$, hemoglobin; 25(OH)D, 25-hydroxyvitamin D; MMSE-KC, Mini-Mental Status Examination in the Korean version of the CERAD assessment packet

† 1 million Korean won = USD 900

$\star P<0.05$ 


\begin{tabular}{|c|c|c|c|}
\hline \multirow[t]{2}{*}{ Characteristic } & \multirow{2}{*}{$\begin{array}{l}\text { Male } \\
(n=1111)\end{array}$} & \multirow{2}{*}{$\begin{array}{l}\text { Female } \\
(\mathrm{n}=1212)\end{array}$} & \multirow[t]{2}{*}{$P$} \\
\hline & & & \\
\hline Dyslipidemia, n (\%) & $277(24.9)$ & $497(41.0)$ & $<0.001^{*}$ \\
\hline Diabetes mellitus, n (\%) & $274(24.7)$ & $243(20.0)$ & 0.16 \\
\hline Depression, n (\%) & $21(1.9)$ & $44(3.6)$ & 0.11 \\
\hline $\mathrm{OA}, \mathrm{n}(\%)$ & $121(10.9)$ & $392(32.3)$ & $<0.001 *$ \\
\hline Osteoporosis, n (\%) & $34(3.1)$ & $312(90.2)$ & $<0.001^{\star}$ \\
\hline WBC, $10^{9} / L($ mean $\pm S D)$ & $5.87 \pm 1.5$ & $5.77 \pm 1.6$ & $<0.001^{\star}$ \\
\hline $\mathrm{Hb}, \mathrm{mmol} / \mathrm{L}($ mean $\pm \mathrm{SD})$ & $14.11 \pm 1.3$ & $12.87 \pm 1.1$ & $<0.001 *$ \\
\hline 25(OH)D (ng/ml, SD) & $24.83 \pm 9.3$ & $22.63 \pm 10.6$ & $<0.001 *$ \\
\hline MMSE-KC (mean \pm SD) & $26.28 \pm 2.8$ & $25.32 \pm 3.3$ & $<0.001^{*}$ \\
\hline \multicolumn{4}{|c|}{$\begin{array}{l}\text { Abbreviations: BMI, body mass index; HGS, handgrip strength; ASMI, appendicular skeletal muscle } \\
\text { mass index; SPPB, short physical performance battery; OA, osteoarthritis; WBC, white blood cell; Hb, } \\
\text { hemoglobin; } 25(\mathrm{OH}) \mathrm{D} \text {, 25-hydroxyvitamin D; MMSE-KC, Mini-Mental Status Examination in the Korean } \\
\text { version of the CERAD assessment packet }\end{array}$} \\
\hline \multicolumn{4}{|l|}{ † 1 million Korean won = USD 900} \\
\hline$\star P<0.05$ & & & \\
\hline
\end{tabular}

Table 2 shows a comparison of the non-faller and faller groups according to the variables of the sarcopenia definition, physical performance parameters, and sex. Among men, the faller group had lower HGS, walking speed, and SPPB score and a higher proportion of patients with severe sarcopenia than the non-faller group. Among women, the faller group had lower HGS, ASMI, walking speed, and SPPB scores than the non-faller group. 
Table 2

Sarcopenia parameters of the non-faller group and faller group according to sex

\begin{tabular}{|c|c|c|c|c|c|c|}
\hline \multirow[t]{2}{*}{ Characteristic } & \multicolumn{2}{|l|}{ Male } & \multirow[t]{2}{*}{$P$} & \multicolumn{2}{|l|}{ Female } & \multirow[t]{2}{*}{$P$} \\
\hline & $\begin{array}{l}\text { Non-faller ( } \mathrm{n} \\
=935)\end{array}$ & $\begin{array}{l}\text { Faller (n } \\
=176)\end{array}$ & & $\begin{array}{l}\text { Non-faller } \\
(n=919)\end{array}$ & $\begin{array}{l}\text { Faller (n } \\
=293)\end{array}$ & \\
\hline HGS, kg (mean \pm SD $)$ & $32.6 \pm 5.8$ & $30.5 \pm 5.8$ & $\dot{0} .001 *$ & $21.3 \pm 3.9$ & $20.7 \pm 3.8$ & $0.044^{\star}$ \\
\hline $\begin{array}{l}\text { ASMI, } \mathrm{kg} / \mathrm{m}^{2}(\text { mean } \pm \\
\mathrm{SD})\end{array}$ & $7.04 \pm 0.8$ & $6.97 \pm 0.8$ & 0.297 & $5.86 \pm 0.7$ & $5.74 \pm 0.7$ & $0.014 *$ \\
\hline $\begin{array}{l}\text { Walking speed, } \mathrm{m} / \mathrm{s} \\
\text { (mean } \pm \text { SD) }\end{array}$ & $1.17 \pm 0.3$ & $1.08 \pm 0.3$ & $\dot{0} .001 *$ & $1.09 \pm 0.2$ & $1.03 \pm 0.2$ & $<0.001 *$ \\
\hline SPPB $($ mean \pm SD $)$ & $11.2 \pm 1.2$ & $10.6 \pm 1.6$ & $<.001 *$ & $10.6 \pm 1.4$ & $10.3 \pm 1.8$ & $0.013^{*}$ \\
\hline Sarcopenia $^{\dagger}(n, \%)$ & $135(14.4)$ & $34(19.3)$ & 0.098 & $67(7.3)$ & $25(8.5)$ & 0.485 \\
\hline $\begin{array}{l}\text { severe Sarcopenia§ }(\mathrm{n}, \\
\%)\end{array}$ & $29(3.1)$ & $7(8.5)$ & $0.001 *$ & $32(3.5)$ & $7(2.4)$ & 0.356 \\
\hline \multicolumn{7}{|c|}{$\begin{array}{l}\text { Abbreviations: HGS, hand grip strength; ASMI, appendicular skeletal muscle mass index; SPPB, short } \\
\text { physical performance battery }\end{array}$} \\
\hline \multicolumn{7}{|c|}{$\begin{array}{l}\text { † Sarcopenia: Low HGS (<28 kg for men and }<18 \mathrm{~kg} \text { for women) and low ASMI }(<7.0 \mathrm{~kg} / \mathrm{m} \text { for men } \\
\text { and }<5.4 \mathrm{~kg} / \mathrm{m} \text { for women) }\end{array}$} \\
\hline \multicolumn{7}{|c|}{$\begin{array}{l}\text { §Severe sarcopenia: all sarcopenia criteria and low physical performance (SPPB score } \leq 9 \text { for both } \\
\text { sexes) }\end{array}$} \\
\hline \multicolumn{7}{|l|}{$\star P<0.05$} \\
\hline
\end{tabular}

Table 3 shows the unadjusted and fully adjusted logistic regression analysis results of sarcopenia definition and other parameters defined by the AWGS according to a history of fall. The male faller group had a significantly low HGS (OR $=1.670,95 \%$ confidence interval $[\mathrm{Cl}]=1.171-2.382)$, SPPB score $(\mathrm{OR}=$ $2.439,95 \% \mathrm{Cl}=1.600-3.718)$, and $4-\mathrm{m}$ walking speed $(\mathrm{OR}=2.528,95 \% \mathrm{Cl}=1.802-3.547)$ in the unadjusted model. In contrast, the female faller group had a significantly low $A S M I(O R=1.392,95 \% \mathrm{Cl}=$ $1.048-1.850)$, SPPB score $(\mathrm{OR}=1.505,95 \% \mathrm{Cl}=1.110-2.042)$, and 4 -m walking speed $(\mathrm{OR}=1.439,95 \%$ $\mathrm{Cl}=1.101-1.881)$ in the unadjusted model. Meanwhile, the male faller group had a significantly low HGS $(\mathrm{OR}=1.508,95 \% \mathrm{Cl}=1.028-2.211)$, low SPPB $(\mathrm{OR}=2.068,95 \% \mathrm{Cl}=1.308-3.271)$, low $4-\mathrm{m}$ walking speed $(\mathrm{OR}=3.499,95 \% \mathrm{Cl}=2.044-5.991)$, and severe sarcopenia $(\mathrm{OR}=2.417,95 \% \mathrm{Cl}=1.224-4.771)$ in the fully adjusted model. However, the female faller group only had a low ASMI in the fully adjusted model $(\mathrm{OR}=1.419,95 \% \mathrm{Cl}=1.058-1.903)$. Other physical performance parameters, including low SPPB score and 4-m walking speed, were attenuated when the confounding factors were considered. 
Table 3

Logistic regression analysis of sarcopenia parameters according to the vitamin B12 level

\section{Characteristic}

Unadjusted model

\begin{tabular}{|ll}
\hline Male & Female \\
\hline OR $(95 \% \mathrm{Cl})$ & OR $(95 \% \mathrm{Cl})$ \\
\hline
\end{tabular}

Fully adjusted model

Male Female

OR $(95 \% \mathrm{Cl}) \quad$ OR $(95 \% \mathrm{Cl})$

$1.109(0.803-$

$1.531)$

$1.508(1.028-$

$2.211)^{\star}$

$0.941(0.669-$

1.324)
Low $\mathrm{HGS}^{\dagger}$

$1.670(1.171-$
$2.382)^{\star}$

Muscle mass

$0.845(0.611-$

1.167)

$1.392(1.048-$
$1.850)^{*}$

$0.828(0.593-$

$1.474(1.077-$

Low $\mathrm{ASMI}^{\dagger}$

Physical
performance
Low SPPB $^{+}$

\section{Low 4-m walking}

speed $^{\dagger}$

$2.439(1.600-$
$3.718)^{\star}$

$1.505(1.110-$

$2.042)^{*}$

$2.159(1.365-$
$3.414)^{\star}$

$2.016)^{*}$

\begin{tabular}{|c|c|c|c|c|}
\hline Sarcopenia $^{+\dagger}$ & $\begin{array}{l}1.419(0.936- \\
2.151)\end{array}$ & $\begin{array}{l}1.186(0.734- \\
1.916)\end{array}$ & $\begin{array}{l}1.309(0.828- \\
2.071)\end{array}$ & $\begin{array}{l}1.041(0.629- \\
1.722)\end{array}$ \\
\hline nia & $\begin{array}{l}2.911(1.526- \\
5.550)^{\star}\end{array}$ & $\begin{array}{l}0.678(0.296- \\
1.554)\end{array}$ & $\begin{array}{l}2.538(1.274- \\
5.056)^{\star}\end{array}$ & $\begin{array}{l}0.542(0.231- \\
1.272)\end{array}$ \\
\hline
\end{tabular}

Abbreviations: OR, odds ratio; $\mathrm{Cl}$, confidence interval; $\mathrm{HGS}$, hand grip strength; $\mathrm{ASMI}$, appendicular skeletal muscle mass index; SPPB, short physical performance battery

The fully adjusted model was adjusted for age, depression, osteoarthritis, osteoporosis, diabetes mellitus, hypertension, location of residence, family group, vitamin D level, number of medications, and body mass index.

+ Low HGS, $<28 \mathrm{~kg}$ for men and $<18 \mathrm{~kg}$ for women; low ASMl, $<7.0 \mathrm{~kg} / \mathrm{m}$ for men and $<5.4 \mathrm{~kg} / \mathrm{m}$ for women; low SPPB score, $\leq 9$ for both sexes; Low $4-\mathrm{m}$ walking speed, $<1 \mathrm{~m} / \mathrm{s}$ for both sexes

${ }^{+\dagger}$ Sarcopenia: Low HGS ( $<28 \mathrm{~kg}$ for men and $<18 \mathrm{~kg}$ for women) and low ASMI ( $<7.0 \mathrm{~kg} / \mathrm{m}$ for men and $<5.4 \mathrm{~kg} / \mathrm{m}$ for women)

§Severe sarcopenia: all sarcopenia criteria and low physical performance (SPPB score $\leq 9$ for both sexes)

$* \mathrm{P}<0.05$

$\star * \mathrm{P}<0.01$

\section{Discussion}


We investigated the associations between sarcopenia definitions and their individual components and the incidence of falls based on sex differences. In men, low HGS (which represents muscle strength) and low physical performance parameters such as low SPPB score and low walking speed had an impact on a history of falls; in women, only a low ASMI (which indicates muscle mass) had an effect. The definition of sarcopenia according to the AWGS, which is characterized by low muscle strength and muscle mass, did not show a significant relationship with a history of falls in either sex group. Severe sarcopenia, defined as sarcopenia plus low physical performance, showed a significant relationship with a history of falls. These results can be explained by the different risk of falls in men and women.

The risk factors for sarcopenia are similar to those for age-related conditions [15]. Since the risk factors for falls are associated with those for sarcopenia, the correlation between falls and sarcopenia has been studied. However, the association between sarcopenia and falls is controversial. Several previous studies have evaluated of the association between sarcopenia, defined by EWGSOP diagnostic criteria, and falls. In an Italian study, Landi et al. studied the relationship between sarcopenia and the 2-year risk of falls. In a total of 250 patients aged $\geq 80$ years, falls were highly prevalent among older persons with sarcopenia regardless of sex differences $(\mathrm{OR}=3.23,95 \% \mathrm{Cl}=1.25-8.29)$ [16]. In another study in Chile conducted in 1,006 community-dwelling participants aged $>60$ years, falls were associated with sarcopenia defined by the EWGSOP [17]. However, other studies have reported a non-significant association between sarcopenia and falls. In a Columbian study conducted in 534 participants (mean age $=74.4$, SD $=8.2$ ), falls that occurred in the previous year showed no significant relationship with sarcopenia [18]. In a previous UK study conducted in 286 participants with a mean age of 76.1 years, no significant associations were observed between sarcopenia and a history of falls [19]. Previous studies mainly defined sarcopenia based on the ESGSOP criteria; however, due to the differences in body composition between Asians and Caucasians, sarcopenia defined by the AWGS was found to be more relevant in Asians. Even with the same BMI, Asians have a higher body fat percentage, lower skeletal muscle mass, and prominent abdominal obesity than Caucasians [20]. Only one study on sarcopenia, defined by the AWGS, and falls was examined. A 2-year prospective Japanese study conducted in 162 individuals showed a significant risk of falls in the sarcopenia group in the adjusted logistic regression analysis $(\mathrm{OR}=7.68,95 \% \mathrm{Cl}=1.41-$ 41.77) [21]. However, this study had certain limitations-it had a small sample size, 61 of the 223 participants withdrew from the study, and the study was conducted in rural areas with a limited population.

In this study, no correlation was found between falls and the overall sarcopenia definition, but a relationship was found between falls and the sarcopenia component parameters. In men, a correlation was found between low muscle strength, low physical performance, and a history of falls. These results were similar to those reported a previous study. In a recent study conducted in Taiwan, low HGS was associated with a history of falls in both sexes [22]. Our study also showed the same results when the average HGS of fallers was compared to thar of non-fallers. However, when the cutoff value of the AWGS was set, low HGS increased the incidence of falls in men, but not in women. Although a mean difference in muscle strength was observed between the faller group and non-faller group, only men showed a significant difference when the muscle strength decreased, which was one of the criteria for diagnosing 
sarcopenia. In both sexes, the faller group showed lower mean SPPB and walking speed values, which indicate physical function; this finding is consistent with that reported by previous studies [23]. When compared by AWGS criteria, it is still correlated in the unadjusted logistic regression analysis. However, in women, attenuation was observed in the fully adjusted regression analysis. Low physical function is thought to be an important risk factor for falls in men; in women, other covariates have a greater effect on a history of falls.

In this study, the faller and non-faller groups showed sex differences in terms of muscle mass. In men, a significant difference was observed in ASMI between the faller group and non-faller group, but a lower ASMI was observed in the female faller group. Moreover, a significant association was observed in women in the unadjusted and fully adjusted logistic regression analyses. This is thought to be due to the sex differences in body composition [24]. Women are more strongly affected by alterations in body composition as they have a high body fat percentage and low muscle mass, which leads to the differences in physical performance and balance [25]. In a previous study, Walters et al. reported that a low ASMI was associated with balance deficit and fall incidence in women, but no association was found in men [26]. This finding is consistent with the results of our study, and the sex-related differences in body composition affect balance and the risk of falls. This difference between men and women can be explained by the difference in body composition and is more strongly associated with the lack of balance due to low physical performance in men. In contrast, the fat mass and muscle mass in women are more strongly associated with a lack of balance.

This study has several limitations. This study was cross-sectional in nature. However, this study is considered significant as it is the first large cohort study with $>2,000$ participants to examine the relationship between sarcopenia, defined by the AWGS, and falls. Further prospective studies and randomized controlled studies are warranted to confirm our findings. Second, we used a self-reported questionnaire, which might have led to a possible recall bias of retrospective individual memories. If falls occur during old age, it is considered as a serious event; hence, details of the a history of falls might be accurately remembered. However, the falls possibly occurred $\geq 1$ year or more before the patient developed sarcopenia. Third, 4-m walking speed was used as a physical performance parameter instead of 6-m walking speed. Because the reliability of walking speed increases with gait distance, the result could be affected [27]. Finally, since this study was conducted in older adults, which is one of the component parameters of sarcopenia, the results of physical performance tests may vary depending on the patient's fatigue status or body condition. Hence, our results may not be considered generalizable. This problem has also been addressed in other geriatric studies.

\section{Conclusion}

This is the first large cross-sectional cohort study to examine sarcopenia, defined by the AWGS, and its correlation with falls in older Korean population. Our results showed that the incidence of falls did not increase in the group with sarcopenia defined by the AWGS. In this study, we found that sex differences in each sarcopenia component affect the history of falls. Among sarcopenia components, low muscle 
strength, low physical performance in men, and low muscle mass in women were significant risk factors. Therefore, when studying fall risk in old age, sex differences in body composition and physical function should be considered.

\section{Declarations}

\section{Ethics approval and consent to participate}

The KFACS study protocol was approved by the Institutional Review Board (IRB) of the Clinical Research Ethics Committee of Kyung-Hee University Medical Center (IRB no. 2015-12-103), and all participants provided written informed consent. The study was conducted in accordance with the ethical standards of the Declaration of Helsinki.

\section{Consent for publication}

There are no details of individual participants in the manuscript.

\section{Availability of data and materials}

All the cohort data that support the findings of this study are available from the KFACS and open to all researchers on reasonable research requests. All published articles and news articles using the KFACS database, data provision manuals, and contact information are available on the KFACS website (http://www.kfacs.kr).

\section{Competing interests}

The authors declare that they have no competing interests.

\section{Funding}

This study was supported by a grant from the Korea Health Technology R\&D Project through the Korean Health Industry Development Institute, funded by the Ministry of Health \& Welfare, Republic of Korea (grant numbers: HI15C3153 and HI15C3207).

\section{Authors' contributions}

YS and CWW were involved in the design of this study. YS and CWW contributed to the analyses and writing of the manuscript. YS and CWW reviewed the study and performed significant editing of the manuscript. The authors have read and approved the final manuscript.

\section{Acknowledgments}


The authors are grateful to the study participants and the staff of the Korean Frailty and Aging Cohort Study, which was the primary source of data used in our study. We would like to thank Editage (www.editage.co.kr) for providing English language editing assistance.

\section{References}

1. Tinetti ME: Preventing falls in elderly persons. New England journal of medicine 2003, 348(1):42-49.

2. Berry SD, Miller RR: Falls: epidemiology, pathophysiology, and relationship to fracture. Current osteoporosis reports 2008, 6(4):149-154.

3. Pfortmueller C, Lindner G, Exadaktylos A: Reducing fall risk in the elderly: risk factors and fall prevention, a systematic review. Minerva Med 2014, 105(4):275-281.

4. O'Loughlin JL, Robitaille Y, Boivin J-F, Suissa S: Incidence of and risk factors for falls and injurious falls among the community-dwelling elderly. American journal of epidemiology 1993, 137(3):342354.

5. Dionyssiotis Y: Analyzing the problem of falls among older people. International journal of general medicine 2012, 5:805.

6. Walston JD: Sarcopenia in older adults. Current opinion in rheumatology 2012, 24(6):623.

7. Santilli V, Bernetti A, Mangone M, Paoloni M: Clinical definition of sarcopenia. Clinical cases in mineral and bone metabolism 2014, 11(3):177.

8. Volpi E, Nazemi R, Fujita S: Muscle tissue changes with aging. Current opinion in clinical nutrition and metabolic care 2004, 7(4):405.

9. Shafiee G, Keshtkar A, Soltani A, Ahadi Z, Larijani B, Heshmat R: Prevalence of sarcopenia in the world: a systematic review and meta-analysis of general population studies. Journal of Diabetes \& Metabolic Disorders 2017, 16(1):1-10.

10. Liguori I, Russo G, Aran L, Bulli G, Curcio F, Della-Morte D, Gargiulo G, Testa G, Cacciatore F, Bonaduce D: Sarcopenia: assessment of disease burden and strategies to improve outcomes. Clinical interventions in aging 2018, 13:913.

11. Al-Aama T: Falls in the elderly: spectrum and prevention. Canadian Family Physician 2011, 57(7):771-776.

12. Cruz-Jentoft AJ, Bahat G, Bauer J, Boirie Y, Bruyère $O$, Cederholm T, Cooper $C$, Landi F, Rolland $Y$, Sayer AA: Sarcopenia: revised European consensus on definition and diagnosis. Age and ageing 2019, 48(1):16-31.

13. Chen L-K, Woo J, Assantachai P, Auyeung T-W, Chou M-Y, lijima K, Jang HC, Kang L, Kim M, Kim S: Asian Working Group for Sarcopenia: 2019 consensus update on sarcopenia diagnosis and treatment. Journal of the American Medical Directors Association 2020, 21(3):300-307. e302.

14. Hilal S, Perna S, Gasparri C, Alalwan TA, Vecchio V, Fossari F, Peroni G, Riva A, Petrangolini G, Rondanelli M: Comparison between Appendicular Skeletal Muscle Index DXA Defined by EWGSOP1 
and 2 versus BIA Tengvall Criteria among Older People Admitted to the Post-Acute Geriatric Care Unit in Italy. Nutrients 2020, 12(6):1818.

15. Grundstrom AC, Guse CE, Layde PM: Risk factors for falls and fall-related injuries in adults $\mathbf{8 5}$ years of age and older. Archives of gerontology and geriatrics 2012, 54(3):421-428.

16. Landi F, Liperoti R, Russo A, Giovannini S, Tosato M, Capoluongo E, Bernabei R, Onder G: Sarcopenia as a risk factor for falls in elderly individuals: results from the ilSIRENTE study. Clinical nutrition 2012, 31(5):652-658.

17. Lera L, Albala C, Sánchez H, Angel B, Hormazabal M, Márquez C, Arroyo P: Prevalence of sarcopenia in community-dwelling Chilean elders according to an adapted version of the European Working Group on Sarcopenia in Older People (EWGSOP) criteria. J Frailty Aging 2017, 6(1):12-17.

18. Benjumea A-M, Curcio C-L, Duque G, Gomez F: Dynapenia and sarcopenia as a risk factor for disability in a falls and fractures clinic in older persons. Open access Macedonian journal of medical sciences 2018, 6(2):344.

19. Clynes M, Edwards M, Buehring B, Dennison E, Binkley N, Cooper C: Definitions of sarcopenia: associations with previous falls and fracture in a population sample. Calcified tissue international 2015, 97(5):445-452.

20. Wulan S, Westerterp K, Plasqui G: Ethnic differences in body composition and the associated metabolic profile: a comparative study between Asians and Caucasians. Maturitas 2010, 65(4):315319.

21. Matsumoto $H$, Tanimura $C$, Tanishima S, Osaki $M$, Noma $H$, Hagino $H$ : Sarcopenia is a risk factor for falling in independently living Japanese older adults: a 2-year prospective cohort study of the GAINA study. Geriatrics \& gerontology international 2017, 17(11):2124-2130.

22. Yang N-P, Hsu N-W, Lin C-H, Chen H-C, Tsao H-M, Lo S-S, Chou P: Relationship between muscle strength and fall episodes among the elderly: the Yilan study, Taiwan. BMC geriatrics 2018, 18(1):17.

23. Veronese N, Bolzetta F, Toffanello ED, Zambon S, De Rui M, Perissinotto E, Coin A, Corti M-C, Baggio G, Crepaldi G: Association between short physical performance battery and falls in older people: the Progetto Veneto Anziani Study. Rejuvenation research 2014, 17(3):276-284.

24. Wells JC: Sexual dimorphism of body composition. Best practice \& research Clinical endocrinology \& metabolism 2007, 21(3):415-430.

25. Valentine RJ, Misic MM, Rosengren KS, Woods JA, Evans EM: Sex impacts the relation between body composition and physical function in older adults. Menopause (New York, NY) 2009, 16(3):518.

26. Waters DL, Qualls C, Cesari M, Rolland Y, Vlietstra L, Vellas B: Relationship of incident falls with balance deficits and body composition in male and female community-dwelling elders. The journal of nutrition, health \& aging 2019, 23(1):9-13.

27. Kim H-j, Park I, joo Lee H, Lee 0 : The reliability and validity of gait speed with different walking pace and distances against general health, physical function, and chronic disease in aged adults. Journal of exercise nutrition \& biochemistry 2016, 20(3):46. 


\section{Abbreviations}

KFACS, Korean Frailty and Aging Cohort study; AWGS, Asian Working Group for Sarcopenia; SPPB, short physical performance battery; HGS, hand grip strength; ASMI, appendicular skeletal muscle mass index; BMI, body mass index; EWGSOP, European Working Group on Sarcopenia in Older People; OR, odds ratio; $\mathrm{Cl}$, confidence interval

\section{Figures}

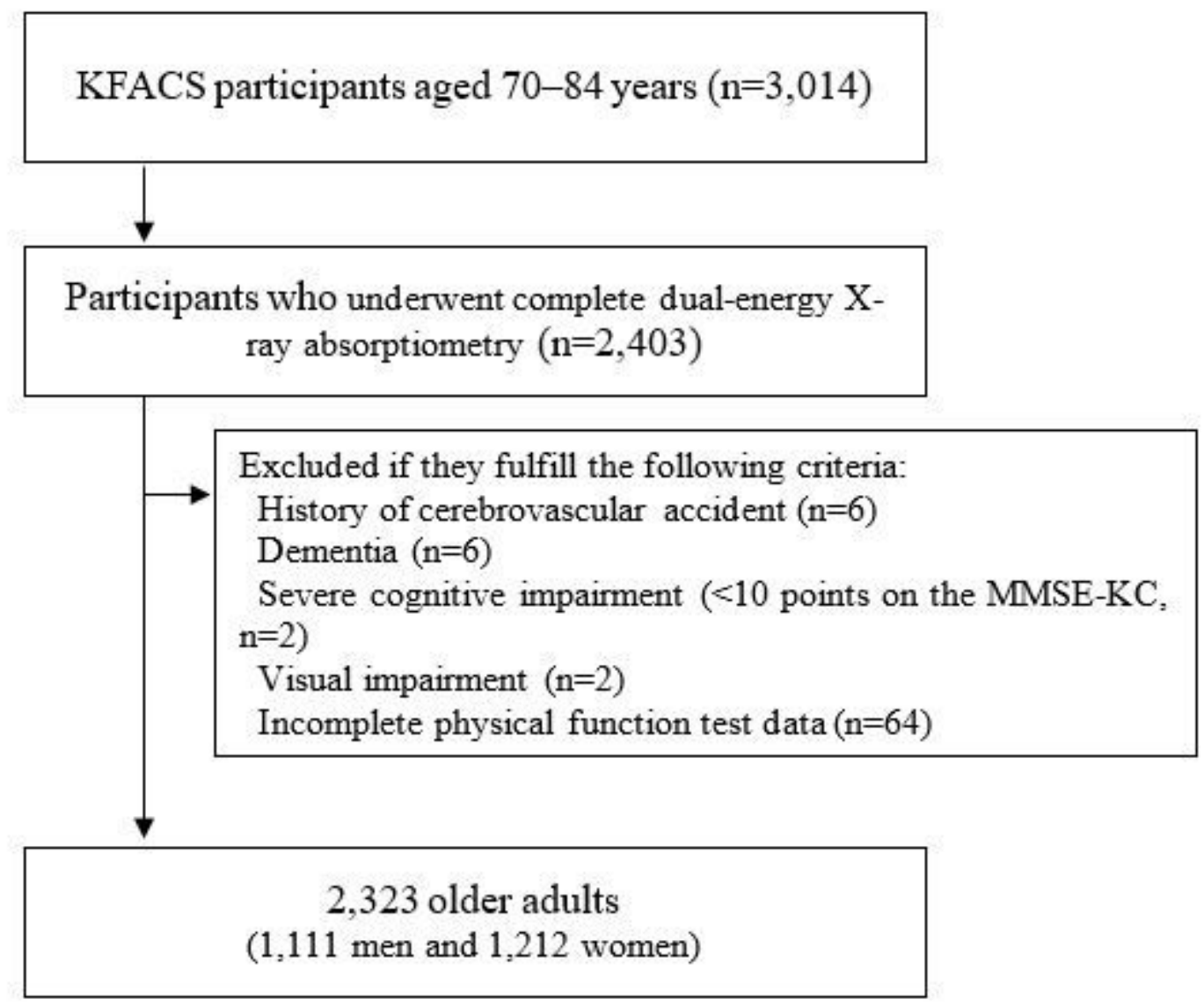

Figure 1

Flow chart of the participant recruitment process KFACS, Korean Frailty and Aging Cohort Study; MMSE$\mathrm{KC}$, Mini-Mental Status Examination in the Korean version of the CERAD assessment packet 